\title{
Where to vacation? An agent-based approach to modelling tourist decision-making process
}

\author{
Inês Boavida-Portugal, Carlos Cardoso Ferreira \& Jorge Rocha
}

To cite this article: Inês Boavida-Portugal, Carlos Cardoso Ferreira \& Jorge Rocha (2017) Where to vacation? An agent-based approach to modelling tourist decision-making process, Current Issues in Tourism, 20:15, 1557-1574, DOI: 10.1080/13683500.2015.1041880

To link to this article: https://doi.org/10.1080/13683500.2015.1041880

\section{Published online: 26 May 2015.}

Submit your article to this journal $\sqsubset$

Џll Article views: 944

Q View related articles $\asymp$

View Crossmark data $\nearrow$

4

Citing articles: 8 View citing articles $\square$ 


\title{
CURRENT ISSUES IN METHOD AND PRACTICE
}

\section{Where to vacation? An agent-based approach to modelling tourist decision-making process}

\author{
Inês Boavida-Portugal ${ }^{*}$, Carlos Cardoso Ferreira and Jorge Rocha \\ Institute of Geography and Spatial Planning, Universidade de Lisboa, Lisbon, Portugal
}

(Received 30 June 2014; accepted 10 April 2015)

\begin{abstract}
Agent-based models (ABMs) are becoming more relevant in social simulation due to the potential to model complex phenomena that emerge from individual interactions. In tourism research, complexity is a subject of growing interest and researchers start to analyse the tourism system as a complex phenomenon. However, there is little application of ABMs as a tool to explore and predict tourism patterns. The purpose of the paper is to develop an ABM that increases knowledge in tourism research by (i) considering the complexity of tourism phenomenon, (ii) providing tools to explore the complex relations between system components and (iii) giving insights on the functioning of the system and the tourist decision-making process. A theoretical $\mathrm{ABM}$ is developed to improve knowledge on tourist decision-making in the selection of a destination to vacation. Tourists' behaviour, such as individual motivation, and social network influence in the vacation decision-making process are hereby discussed.
\end{abstract}

Keywords: agent-based models; complexity; tourism system; tourist behaviour; decision-making process; simulation

\section{Introduction}

Identifying the underlying mechanisms of tourism system is a fundamental challenge for tourism research and holds implications for planning and management (Farrell \& Twining-Ward, 2004; Mill \& Morrison, 2009). In general, traditional tourism research has focused on aspects of behaviour and development patterns exhibiting order, linearity and equilibrium. Based on the Newtonian paradigm of scientific inquiry, systems are understood to be highly dependent on initial conditions that explain the future outcomes (McKercher, 1999). The system is dissected into its components and behaviours are interpreted individually, assuming clock-like relationships. Tourism forecasting has been based in techniques that compile data from past phenomenon in order to extrapolate future predictions, assuming that the dynamics of the system will be maintained (Baggio \& Klobas, 2011). These kinds of techniques assume that the system is predictable and operates in a linear way. However, systems characterized by disorder, instability and non-linearity are predominant. In such cases possible key events can be filtered out of research due to unpredictability and noise as a way to produce more accurate predictions (Faulkner, 2000).

Most tourism models recognize the complex nature of the tourism system. Some early studies such as Gunn (1988), Pearce (1989) and Leiper (1990) sustained that the system as a whole could be understood by identifying and deconstructing the components and the

*Corresponding author. Email: iboavida-portugal@campus.ul.pt 
relationships between them. By understanding how each component works, knowledge of the whole system is believed to emerge. These models conceptualize tourism as a system formed by interconnected and interdependent parts, characterized by discrete components such as market, travel, destination and marketing, but failed to test the mechanisms that drive the individual interactions. There is an implicit assumption that tourism is a linear, deterministic activity that can be controlled and predicted by analysing the sum of its component parts (McKercher, 1999). In fact, this reductionist approach advocates that the system is just the sum of its components.

The current work reports a complex systems approach to the comprehensive investigation of the tourist decision-making process in the selection of a destination. Where and why tourists decide to vacation form the main objective of this agent-based model (ABM). The work starts by exploring complexity and ABM applications in tourism and the need to adopt such approaches. Then an ABM is developed as a proof of concept to enhance the understanding of the tourist decision-making process. The tourist decision ABM considers (i) individual motivations, that is, behaviour in response to basic need or preferences; (ii) rationality based on if-then rules; (iii) human emotions and satisfaction and (iv) the influence of the social network in which the individual is inserted in the destinations choice (Kennedy, 2012). For this purpose, in this paper, two scenario experiments were developed to explore how changes in awareness that a tourist has for a destination and how tourist individual preferences change the destination selection process.

\section{Complexity in tourism}

Recently, the understanding of the functioning of the tourism system has taken a rather different perspective, with the application of complex system and chaos theories in tourism research. Recognition that tourism is a complex phenomenon is currently discussed within academic literature (Baggio, 2008; Baggio \& Sainaghi, 2011; Faulkner \& Russell, 1997; Faulkner, Russell, Moscardo, \& Laws, 2001; Russell \& Faulkner, 1999). Complexity and chaos are acknowledgeable concepts in the tourism system and many scholars believe these concepts are able to give a better understanding of the dynamical behaviours that define the system.

Complexity and chaos approaches address tourism as being composed of interconnected and interdependent components which behave in a non-linear, self-organizing, on-the-edge-of-chaos way. When incorporating features that in a reductionist approach would be considered as noise, one begins to grasp the complexity of the interactions between components and understand that the system is to be analysed as a whole.

The interactions are mathematically non-linear, that is, a change in one component may not have a proportional effect in other components. In fact, a very small change can produce large effects on the system, addressing Lorenz's butterfly effect theory which defended the feasibility that a wing flapping of a butterfly in Beijing could initiate a series of effects, resulting in a cyclone in Florida (Lorenz, 2000). On the contrary, effects thought to have great impact may actually produce hardly any changes on the overall system (Horn, 2002). Therefore, emergent properties arise in larger scales driven by changes at lower ones (Batty, 1995; Wu \& Webster, 1998). Emergent properties occur when local interactions produce phenomena that are more than a simple sum of the components of the system (Epstein, 1999), justifying the need to analyse it as a whole. The Schelling (1969) model is a classic example of how emergent behaviour of class segregation can result from individual preferences (local scale) for neighbour type. 
Systems are dynamic and often change from an equilibrium state to a chaotic one. Therefore no state of equilibrium is permanent. Tourism itself is a phenomenon characterized by a high level of dynamism. Faulkner (2000) argued that in the tourism system, equilibrium and chaotic states often shift due to changes in system components. The author points out changes in socio-economic environment affecting tourism demand; changes in natural environment jeopardizing sustainability of tourism development; and technological innovations that create new market opportunities, among other initiatives that potentially affect destination competitiveness. According to Johnson and Sieber (2010), the success or failure of destinations can be considered as a pattern that emerges from the multiscaled interactions of tourists, destinations and communities. Thus, even shifting between stability and instability, systems have the ability to self-organize through the adaptation of its components and their relationships to a changing environment (Bertalanffy, 1968; Lewin, 1999).

\section{ABMs and tourism research}

Over the last decade, ABMs registered increasing applications in human behaviour modelling. Despite the growing interest in complexity and chaos theory in tourism research (Johnson \& Sieber, 2009; McDonald, 2009; McKercher, 1999; Scott, Cooper, \& Baggio, 2008; Zahra \& Ryan, 2007), there has been little empirical research on the subject. The relationship between ABMs and complexity is reciprocally favourable. On one hand, complexity theory provides the theoretical background and concepts for ABMs, while advances in ABMs provide tools to explore and represent complex phenomena (Manson, Sun, \& Bonsal, 2012). ABMs allow for the representation of a system from a bottom-up perspective, designing the components at an individual level through entities known as agents. These are modelled according to a predetermined set of rules that describe their key attributes and behaviours. By modelling at an individual level, it is possible to incorporate the diversity of behaviours, attributes and interactions inside the system by adopting a bottomup, agent-by-agent and interaction-by-interaction approach (Macal \& North, 2010). Through this approach one can represent emergent properties that usually characterize systems comprising components that (i) display non-linear relations, (ii) have thresholds, (iii) possess memory, (iv) are path dependent and (v) have learning and adaptation capabilities (Bonabeau, 2002).

Agents can represent any scale, from single individuals to institutions. In the tourism system, agents can represent a single tourist, destination, stakeholder or heterogeneous groups of individuals with different characteristics (Johnson \& Sieber, 2011). The relationships between agents are specified, linking agents to other agents and to the environment. The environment serves as the support for the simulation and is represented by a cellular model that can contain geographical information. An ABM develops over a simulated time frame (or time steps) in which agents behave according to a set of defined if-then rules (Bonabeau, 2002). This requires for an adaptation of the system through feedback loops. As Baggio and Baggio (2009) showed in their simulation to verify the relationships between the attractiveness of a tourist destination and tourist arrivals, agents tend to go where their personal criteria are fulfilled. At every time step, agents act according to the defined rules repeating the process until the selection of the best destination is true, constituting an adaptive behaviour. Usually the simulation stops when the criteria of the rules are met.

ABMs can be thought of as a social virtual laboratory as they provide a tool to model emergent complexity, where the key attributes of the agents and the environment can be 
simulated over multiple time steps, allowing an examination of possible futures or pasts of a system. Hence ABMs provide a tool to "think with", assisting in the decision-making process (Batty, Crooks, See, \& Heppenstall, 2012).

When using an ABM, one has to make a commitment between the purpose of the model and its precision, that is, the type of data and knowledge that is required. Throughout the literature the authors point out two categories for ABM utility. Although the nomenclature is not consentaneous in all cases, on one hand we have the theoretical or exploratory models, and on the other hand empirical or predictive models (Batty, 2008; Couclelis, 2005; Crooks, Castle, \& Batty, 2008; Parker, Manson, Janssen, Hoffmann, \& Deadman, 2003).

Theoretical models enable the understanding of how relationships between components of the system work over time. These models are hypothetical and proof of concept oriented, providing the exploration of theories, behaviours and patterns within a simulation environment (Johnson \& Sieber, 2009). Theoretical models are built for illustrative purposes, for example, not recurring to real-world data, and are often called as toy models. These models can generate insights about theory and thinking on complex systems. However, their validation is difficult and therefore raises issues in perceiving if the knowledge produced is relevant (Crooks et al., 2008).

Empirical models are based on real-world data. The selection of data to incorporate in the model is a thorough process in order to accurately describe and represent the system components. By adding redundant information, the empirical model can be over-fitted, that is, the calibration is constrained to existing data making the model too specific to analyse alternative system simulations (Castle \& Crooks, 2006). In tourism research, variables such as visitor counts, origin/destination, tourism establishments, accommodation capacity and tourist choice preferences, among others, could be incorporated in an empirical model (Zellner, 2008).

By accounting for individual heterogeneity and multiple relationships between agents, ABMs can reproduce feedback processes, providing a tool to determine how the system has evolved to the present state and to test future simulations. Therefore, the choice of adopting a theoretical or empirical approach in an ABM is dependent on the purpose of the model and its precision. The availability of data and knowledge required for modelling also influences the choice of the approach. In this paper we present a theoretical proof-of-concept $\mathrm{ABM}$ which main purpose is to enrich the understanding of the tourist decision-making process, through controlled computational simulations.

\section{Developing a tourist decision-making ABM}

Developing an ABM, as in any model, is based on creating simplifications of real-world problems. By modelling tourists' decision-making process, the aim is to describe in simple terms a complex phenomenon, based on incomplete knowledge and data. This process is defined by simple if-then rules that replicate tourists' behaviour and pattern formation. The goal of the model, the data available and the knowledge of system dynamics set the boundaries on the ABM detail. In a theoretical model there are little limitations to what data and parameters can be included in the model, while empirical models should be based on reliable data and have solid theoretical understanding of the system. Either way, there is a model-building process in ABM that can be represented in several stages: (i) the conceptualization of the system that leads to the definition of purpose of the model; (ii) the definition of variables, parameters and data to be used; (iii) the setting of if-then rules which translate behaviours and (iv) finally, the coding and application of the model. 
In order to understand where and why tourists decide to vacation requires a knowledge on tourists behaviour, which is determined by factors such as motivation, perception, learning and attitudes, which are themselves influenced by personality, culture and society (Mill \& Morrison, 2009). There is the hypothesis that people travel if they learn that travel will help satisfy various needs and wants considered important to them, within constrains such as time and money. Dann (1977) noted two stages in a vacation decision: (a) push factors such as motivation and desire to travel, that is, factors internal to the individual; and (b) pull factors which are external to the individual. Tourists travel to a destination if the benefits offered by the destination (pull factors) are perceived to satisfy their needs (Goossens, 2000). The tourists demonstrate loyalty to the destination that satisfies their needs by coming back and recommending the destination to others (Yoon \& Uysal, 2005). The model of buyer behaviour in tourism research suggests that the stimuli internal to the individual relating to needs and wants are the beginning of the decision-making process (Cohen, Prayag, \& Moital, 2014). Then, based on external stimuli the potential traveller is aware (or not) of possible destinations which are evaluated. The tourist weights various alternatives against a list of criteria considered important and likely to satisfy individual needs. The alternative destination chosen will be the one that best meets the motivation. If a destination is considered as an alternative depends greatly on the satisfaction of previous individual experiences the tourist had in that same destination. The level of satisfaction is a function of what is expected and the actual experience. As the satisfaction level increases, the number of alternatives considered in the next vacation decreases. The more an individual is pleased with a vacation choice, the higher that choice will be placed on the list of alternatives and the more likely the tourist is to come. Tourists make their choice based on previous experiences and also based on specific criteria, such as information taken from commercial or social network (friends and relatives). Finally, the tourist travels to the destination that best meets the criteria defined. As a result of the trip, tourists learn whether or not that experience satisfied the needs and wants previously identified, and if it did, they are likely to return to the same destination next time (Bansal \& Eiselt, 2004).

Taking this theoretical approach, the ABM developed intends to reproduce tourist behaviour in a simple way. Several parameters are incorporated and tested in order to model individual behaviour in the destinations choice. As mentioned earlier, the tourist decision ABM considers (i) individual motivations; (ii) rationality based on if-then rules; (iii) human emotions and satisfaction and (iv) the influence of the social network (Kennedy, 2012).

\section{Functioning of the ABM}

The methodological structure of the ABM building can be seen in Figure 1. According to Gilbert and Troitzsch (2005), an ABM is developed first by abstracting agent behaviour from the target system under study through a parameterization process. The parameters selected aim to represent realistically complex phenomena and can be assigned randomly, from statistical distributions, according to heuristic decision trees, or other methods (Epstein, 1999). The theoretical model is substantiated by the parameters that rely on theoretical and empirical assumptions of the system functioning, which require an understanding of system components and agent behavioural rules. The ABM is then adjusted to real-world dynamics through the process of calibration using gathered statistical data and translating it into parameter thresholds for the business as usual scenario. The model is then run and the resulting simulation is compared to real-world data gathered from the target system. This process leads to the validation (or not) of the ABM. If the model is validated, it is robust 


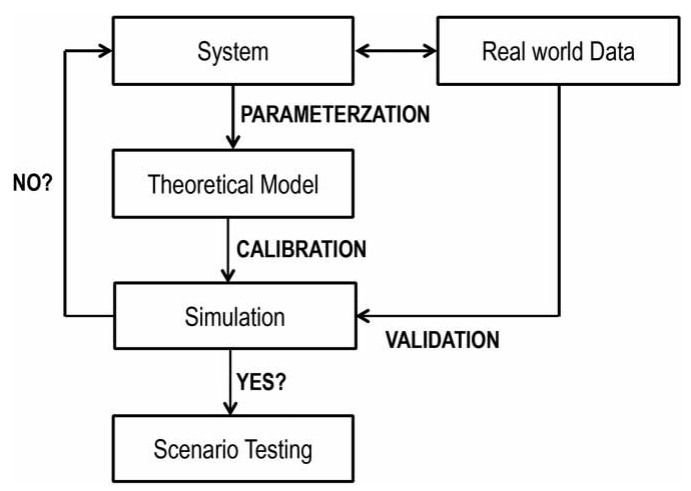

Figure 1. Methodological structure of the ABM.

enough to support scenario testing. If not, the process of ABM building starts from the beginning adjusting and modifying theoretical assumptions and parameters.

This ABM models the decision context of tourists coming from one origin to five destinations. Because geographical data were not incorporated, the model is aspatial and does not incorporate variables such as distance and cost to travel from the origin to the destination. Although this ABM is a toy model, an empirical case study was selected and realworld data were incorporated to allow model calibration and validation. Data for the total number of tourist' arrival and specific weight for each destination were derived from tourism data sets from the National Statistics Institute. The tourist motivation was based on a survey to characterize tourists' types and personal motivation to travel, produced in 2013 by Alentejo Tourism Observatory for the Alentejo Regional Tourism Entity. The attractiveness of the destination for Alentejo coastal area is based on empirical knowledge of the area and on the theoretical main strategic tourism market segments pointed out by Tourism National Strategic Plan (PENT) for the period 2013-2015.

To operationalize the ABM, Alentejo coastal area was selected as the case study. It is a NUTIII located in Alentejo (south of Portugal) with approximately $5300 \mathrm{~km}^{2}$ and 98,000 inhabitants. Typifying a rural region, Alentejo coastal area has great tourism potential and is highlighted in the PENT as a touristic development pole with several strategies outlined to increase the number of tourists and their expenditure. In 2012, the region received 140,000 tourists, mainly Portuguese and Spanish during the summer season. Alentejo coastal area has a set of distinctive assets that provide a very attractive atmosphere, such as climate, pristine beaches, natural and protected areas, gastronomy, golf and proximity to Lisbon. The strategic market segments for the region are sun and sea, golf, food and wine, integrated resorts and residential tourism, mostly vacationed for national and Spanish tourists. However, there are some land-use restraints due to $30 \%$ of total area being covered by natural protected areas. This fact together with the projected increase in tourism supply raises awareness about questions such as the promotion of sustainable tourism development or balancing tourism accommodations development with the preservation of natural landscape characteristics. The knowledge of how tourism demand operates provides insights for a better adjustment between supply and demand, in order to avoid some unsuccessful tourism destination's examples. Some areas of Algarve and south of Spain are examples of inadequate tourism planning and constitute well-known examples of destinations that are nowadays subject of planning intervention for conversion and restructuring of touristic spaces. 


\section{Setting the agents: tourists and destinations}

This ABM represents tourism as the relationships between the destinations and tourists (supply and demand), and tourists among themselves. Thus, there are two types of agents in the ABM, each endowed with different attributes: tourists and destinations. Tourists $(T)$ are mobile agents. They have distinctive profiles that are related to their personal preferences. Each tourist has a personal motivation list $\left(\operatorname{mot}_{T}\right)$ with three key elements: sun and sea, culture and gastronomy-related activities. These elements and the tourist profiles were set up based on the mentioned survey (ERTA, 2013). For each element on the motivation list, the tourist has a value that corresponds to the drive for these activities in a destination; for example, if a tourist wishes sun and sea, type of vacation will have a high value in the sun and sea seeker parameter. The setup function generates three different kinds of tourist agent profiles $(P)$ : sun and sea seeker, culture seeker and gastronomy seeker, defined in Table 1.

Destinations $(D)$ are non-mobile agents and serve as the environment for the simulation. There are five different destinations in Alentejo coastal area: Alcácer do Sal, Grândola, Odemira, Santiago do Cacém and Sines. Each destination has an attraction list $\left(A_{D}\right.$ Table 2) defining the most attracting resources of the destination: sun and sea, culture and gastronomy attractiveness. The values for the attraction are based on the main strategic tourism market segments pointed out by PENT 2013-2015 and by empirical knowledge of the region's tourism characteristics.

Destinations have a specific tourist weight $\left(\right.$ weight $_{D}$ ) which defines the maximum threshold of tourists for each destination and time step in order to make the vacation satisfactory (see Table 3). It is defined by a function of the ratio between the yearly total number of tourist for Alentejo coastal area and the yearly total number of tourists for each destination.

\section{ABM parameterization, calibration and validation}

The outline of the tourist decision-making process developed in the ABM is shown in Figure 2. At each time step, the tourist decision-making process in the selection of a destination depends on two main factors: the individual-level $(I)$ and the social influence (SOC). The individual-level parameters refer to the personal characteristics of tourists and include the priority $(P)$ for a destination, the compatibility $(C)$ between tourists' motivation list $\left(\operatorname{mot}_{T}\right)$ (Table 1$)$ and destination attractiveness $\left(A_{D}\right)$ (Table 2$)$ and the individual satisfaction $(S)$ of the last experience the tourist had in a specific destination.

In the ABM tourists have different priorities for each destination. The priority ranks the destinations according to the urge tourists have to go there, that is, if tourist ${ }_{a}$ goes to destination $_{x}$ in time step 1, the next vacation tourist ${ }_{a}$ is likely to want to go to other destinations rather than $\mathrm{x}$. This way it is created a hierarchy of priorities to visit each destination. At the beginning of the simulation, each tourist agent has the same priority for all the

Table 1. Tourist motivation list.

\begin{tabular}{lccc}
\hline Motivation $\left(\mathrm{mot}_{T}\right)$ & P1 Sun and sea seeker & P2 Culture & P3 Gastronomy seeker \\
\hline Sun and sea & 0.8 & 0.1 & 0.1 \\
Culture & 0.1 & 0.8 & 0.2 \\
Gastronomy & 0.1 & 0.1 & 0.7 \\
\hline
\end{tabular}


Table 2. Destination attraction list.

\begin{tabular}{lccccc}
\hline Attraction $\left(A_{D}\right)$ & Alcácer Sal & Grândola & Santiago Cacém & Sines & Odemira \\
\hline Sun and sea & 0.2 & 0.8 & 0.4 & 0 & 0.8 \\
Culture & 0.2 & 0.1 & 0.4 & 0.8 & 0.1 \\
Gastronomy & 0.6 & 0.1 & 0.2 & 0.2 & 0.1 \\
\hline
\end{tabular}

Table 3. Specific tourist weight of the destinations (2012).

\begin{tabular}{lcc}
\hline Destinations & Tourists/year & Weight $_{D}$ \\
\hline Alcácer Sal & 19,775 & 0.14 \\
Grândola & 48,845 & 0.36 \\
Odemira & 17,532 & 0.13 \\
Santiago Cacém & 23,610 & 0.17 \\
Sines & 27,802 & 0.20 \\
Total & 137,564 & 1.00 \\
\hline
\end{tabular}

destinations, equal to 0.2 for each destination. Every time a tourist visits a destination, the list of priority is updated. The priority for the destination just visited is set to zero because the tourist is not likely to visit the same destination in the next vacation, and the value of the priority before the update is split among the other destinations in equal proportion. The update equation is the following:

$$
\left\{\begin{array}{l}
P_{\text {last }}(t+1)=0 \\
P_{\text {other }}(t+1)=P_{\text {other }}(t)+\frac{P_{\text {last }}(t)}{(\# \text { destinations }-1)}=P_{\text {other }}(t)+\frac{P_{\text {last }}(t)}{4}
\end{array}\right\}
$$

where $P_{\text {last }}$ is the priority value for the destination visited at the last step and $P_{\text {other }}$ is the priority value for all the other destinations (i.e. different from the one visited at the last step).

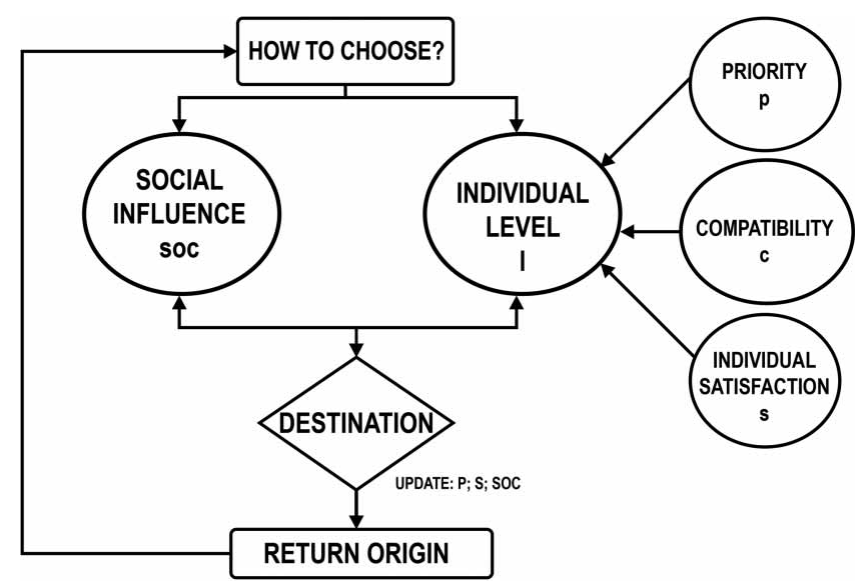

Figure 2. Tourist decision-making process. 
To match tourists' motivation with destination attractiveness, the model incorporates a compatibility parameter $(C)$, computed in the individual level. Tourists with different profiles want to vacation to destinations that match their motivations. For instance, a tourist seeking sun and sea-related activities is more likely to want to vacation in a sun and sea type of destination. Thus the compatibility between the tourists' motivation list $\left(\operatorname{mot}_{T}\right)$ and the characteristics of the destination depicted in the attraction list $\left(A_{D}\right)$ is fundamental in the ABM. The compatibility parameter is computed as

$$
C_{D}=\sum_{i=1}^{n} \operatorname{mot}_{T} \cdot A_{D}
$$

At the beginning of the simulation, each agent has the same satisfaction score $(S)$ for all the destinations (equal to 1 ). When a tourist visits a destination, the satisfaction score is updated based on how many tourists there are in the same destination at the same time, designated as occupancy rate. This parameter is based on the assumption that if the destination is close to the maximum number of tourists it usually receives (based on the specific tourist weight, see Table 3), the destination is crowded and the tourist is less likely to enjoy the vacation experience as much. Thus, the level of satisfaction decreases and tourists are less likely to revisit. The occupancy threshold varies between 0 and 1, computing a ratio between actual tourists and maximum occupancy for each destination; for example, if the value is 1 , there is enough space for all tourists $(100 \%)$, but if the value is 0.2 , only $20 \%$ of tourists will have space and thus a satisfactory experience. There is also a satisfaction weighing parameter $\left(\right.$ weight $\left._{D}\right)$ with a threshold between 0 and 1 , which defines the weight of the last and current vacation; for example, if the value is 0.5 , the satisfaction of the last visit and the satisfaction of the current have the same weight in the computation for the satisfaction parameter.

At each time step, the satisfaction score is updated:

$$
\left\{\begin{array}{l}
S_{D}(0)=1 \\
S_{D}(t)=1-\frac{\# \operatorname{tourists}_{D}}{\text { weight }_{D}} \\
S_{D}(t+1)=w \cdot S_{D}(t)+(1-w) \cdot S_{D}(t-1)
\end{array}\right\} .
$$

To make this parameter more realistic, because the satisfaction of the experience depends on other variables rather than the crowdedness of a destination, some randomness was introduced in this parameter. Therefore, the score of the satisfaction is a function of $S_{D}$ and a random-float value between 0 and 2 .

The social influence is the second factor in the ABM and was incorporated in order to test the formation of networks that result in the "small world" phenomenon. The small world theory, or the concept of six degrees of separation (Milgram, 1967), is based on the idea that a person is only a couple of connections away from any other person in the world. Similarly, tourists are inserted in their own social network (family, friends or co-workers) with whom they share and discuss previous vacation experiences, usually giving an overall score to the destinations (i.e. individual satisfaction). To replicate the small world phenomenon in the ABM was used a network as substrate with an average degree of four, where tourists are connected with their four closest neighbours. Tourists can also be randomly connected with other non-neighbour tourists through an adjustable rewiring probability incorporated in the model. The rewiring probability is a parameter 


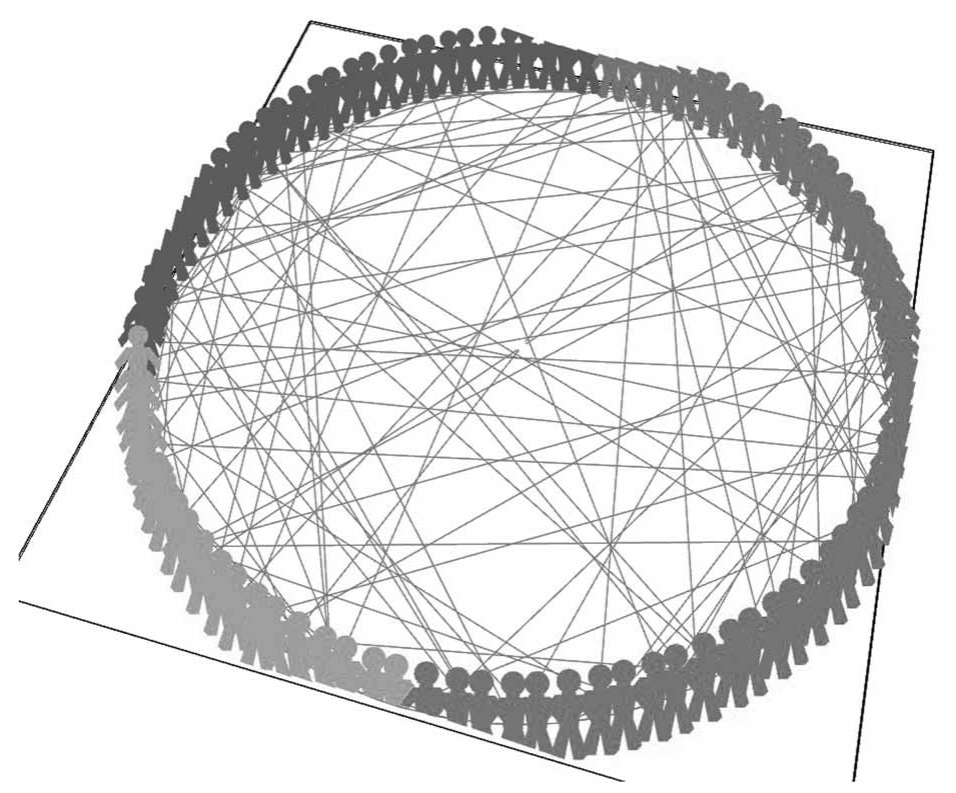

Figure 3. Substrate social network.

of the model with a threshold between 0 and 1 ; that is, if the value is 0.2 , there is a $20 \%$ probability to be connected to another tourist who is not one of the four closest neighbours. This parameter aims to represent social interactions in a more realistic way, in the sense that tourists can share information by other means of communication, such as in an online social network. Thus, a network is created (as shown in Figure 3) incorporating information sharing among tourists about previous vacation experiences.

Tourists share and inform connected neighbours about their individual satisfaction score $\left(S_{D}\right)$ for each destination they visited at previous time steps. The social influence factor is calculated as a mean of connected neighbours' satisfaction score for previous vacations at a destination; that is, if my four connected neighbours share values for satisfaction score for destination $_{x}$ of $\{1 ; 0.2 ; 0.5 ; 0.1\}$, the satisfaction score is computed as 0.45 . The influence that the social network has on tourist destination choice is a model parameter with a threshold between 0 and 1 .

$$
S O C_{D}=\sum_{A \in\{\text { neighbours }\}} S_{A} / \# \text { neighbours. }
$$

There is also a social network weighing parameter that allows the modeller to weight the influence the social network has on the final score $F_{D}$ for the choice of the destination.

By incorporating the social influence factor the ABM replicates the relationships that exist in the real world, for example, if one wishes to go on vacation to destination $_{x}$ is likely to ask the social network their global appreciation of past experiences in that destination and to take it into account in its choice. The social influence is updated every time the individual satisfaction score is updated, that is, at each time step.

The modeller can change parameter thresholds and run different simulations. At each time step the tourist agent computes a final score $F_{D}$ for each destination using 


$$
F_{D}=\mathrm{SOC}_{D} \cdot I_{D}
$$

This way the agent ranks the destinations according to the $F_{D}$ value and chooses to vacation in the one with the highest score.

To test the robustness of the model, the parameter values were calibrated for one destination until the results of the simulation significantly reproduce the real total number of tourists. Afterwards the process of validation was carried out in order to verify if the parameter thresholds defined in the calibration reproduced similar results to the other four destinations of the ABM. The simulation parameter thresholds found to best mirror the real total number of tourists/yearly for the period 2002-2012 were (all parameters on) satisfaction weight 0.60 ; social network rewiring probability 0.50 and social network weight 0.40 , and the occupancy rate was set at 0.55 .

The same thresholds were applied to the simulation of all the destinations and a regression analysis was performed to verify the significance of the results. The results from the validation process show that there is a significant positive correlation between the real total number of tourists in the period 2002-2012 and the simulation results for the same variable, as can be seen by the $R^{2}$ for each destination in Figure 4.
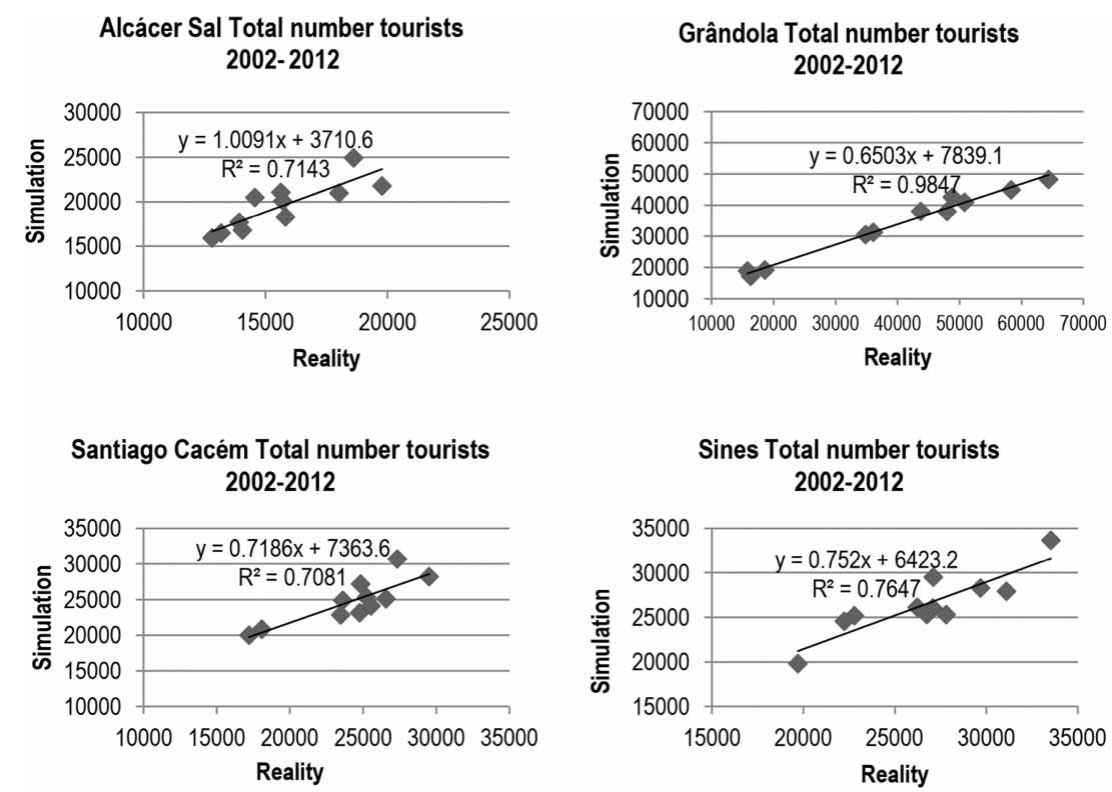

Odemira Total number tourists 2002-2012

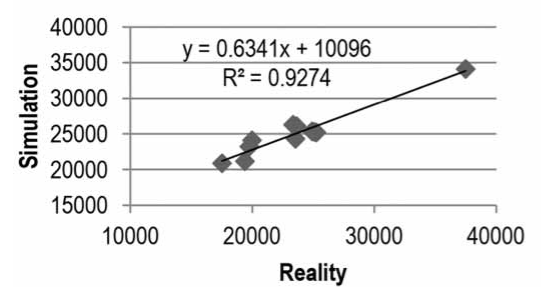

Figure 4. Regression analysis for the real total number of tourists and simulated results for the period 2002-2012. 


\section{Scenario testing}

Different simulations were performed in order to explore the tourist decision-making process. The setup process assigns the values for the tourist motivation and destination attractiveness. Then the user defines the ABM parameter thresholds and the simulation runs 365 time steps (one year period). The ABM was used to generate two different scenarios of tourist visitation. The first scenario investigates the effect of changing tourist awareness of a destination. By giving a wider knowledge to tourist agents about prior experiences in a destination, we increase their awareness. This way we can compare the behaviour of a more aware tourist versus a tourist with less information. The second scenario explores how the individual level drives tourist destination choice, testing how the previous vacation experience and priority for a destination affect the behaviour pattern. These two scenarios are compared to the business as usual scenario (BAU) to evaluate possible effects of awareness increase and individual-level changes.

BAU consists on the simulation carried out in the validation process using the parameter thresholds found to best mirror the real total number of tourists/yearly for the period 20022012. This is the starting point to compare the scenario simulation results. At each time step 430 tourist agents enter the simulation. This value reflects the linear tendency registered for tourist number increase for 2017. The profile for tourists was based on strategic tourism market segments pointed out by PENT 2013-2015 for the region: food and wine, cultural touring and sun and sea. Thus, the tourist agents profile was set as $50 \%$ sun and sea seekers, $20 \%$ culture seekers and $30 \%$ gastronomy seekers. Assuming that BAU illustrates, in simple terms, the reality registered in tourist destination decision-making process, possible scenario simulations are hereby discussed.

\section{Awareness scenario}

The discussed scenario aims to test different levels of awareness that a tourist has for each destination. We assume that the information given by the social network may increase the awareness of a destination. The model is programmed so that when a tourist goes on vacation, he or she shares information with its linked neighbours, exchanging knowledge about previous vacation experiences. This process leads to a more informed choice. Therefore tourists will be more aware if the destination they are considering is likely to meet their satisfaction criteria. The weight the social network has on the tourists' destination choice is a threshold between 0 and 1 (maximum awareness).

The first simulation (Sim1) tested a full awareness scenario in which 1 is the value for social weight. When tourists are well informed they choose to go to the destinations where their neighbours had satisfactory experiences, that is, $34 \%$ of tourists went to Grândola (as shown in Figure 5). This value reflects the tourist decision-making process using BAUdefined parameter thresholds with a maximum social weight. Grândola is the most popular destination due to its high occupancy (0.36), parameter which was defined as accountable for the tourist satisfaction.

However, in the real world we are not always connected to our neighbours. A second simulation was tested $(\operatorname{Sim} 2)$ in which 0.1 is the value for social weight. This simulation results in a more even distribution through the destinations because there is little influence by the social network in choosing a destination.

When comparing BAU with Sim1 and Sim2, we verify significant changes (Table 4). In Sim1 Odemira registers the highest decrease in tourist total number because tourist neighbours share a less positive experience due to low occupancy weight, which 


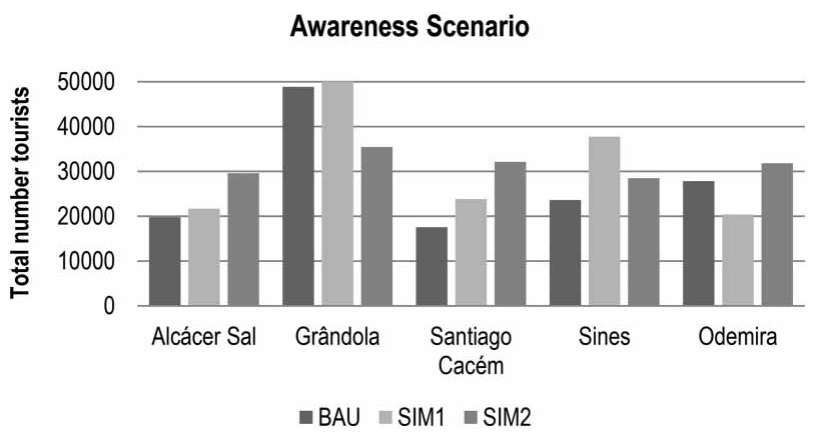

Figure 5. Total number of tourists in the awareness scenario.

means a more crowded experience. Santiago Cacém (26\%) and Sines (37\%) have an increase in tourist numbers because they have a diversified supply base for different tourist profiles and high occupancy weight with a relative low total number of tourists compared to the competitor destinations. Sim2 produces rather different results. Overall, the resulting distribution is more even through the destinations. Grândola registers a $38 \%$ decrease compared to BAU. This fact has to do with the lack of information sharing with neighbours about positive experiences in the destination, since it is the biggest receiving destination in the Alentejo coastal area with the highest occupancy rate. This decrease in total number of tourists in Grândola is accompanied by an increase in all the other destinations. Therefore, in Sim2 the social network is not strong enough to influence significantly tourists' decision-making process. One can speculate that the influence of the social network produces rather different patterns of tourist distribution throughout the destinations.

\section{Individual-level scenario}

In the second ABM scenario, the aim is to explore how the tourist individual-level parameters influence the destination's choice behaviour pattern. The first simulation (Sim3) tested the individual satisfaction impact in the selection of a destination. To do so, the satisfaction parameter is off. This way one can access comparing to BAU the differences the individual satisfaction introduces in the model. There is a quite even distribution of tourists through the destinations (see Figure 6), which means that once the satisfaction of the experience is not a factor of decision, tourists go to the destination that best meets their motivations and priority. $\operatorname{Sim} 3$ can be related to $\operatorname{Sim} 2$ for producing similar results due to the fact that the social influence is closely related to the satisfaction of the experience.

When comparing BAU to $\operatorname{Sim} 3$ (as given in Table 5), it is noticeable a significant decrease in total tourist number in Grândola because the overall experience is the most

Table 4. Change in tourist visitation (\%) between BAU-Sim1 and BAU-Sim2.

\begin{tabular}{lccccr}
\hline Awareness scenario & Alcácer Sal & Grândola & Santiago Cacém & Sines & Odemira \\
\hline Sim1 & $9 \%$ & $9 \%$ & $26 \%$ & $37 \%$ & $-36 \%$ \\
Sim2 & $33 \%$ & $-38 \%$ & $45 \%$ & $17 \%$ & $13 \%$ \\
\hline
\end{tabular}




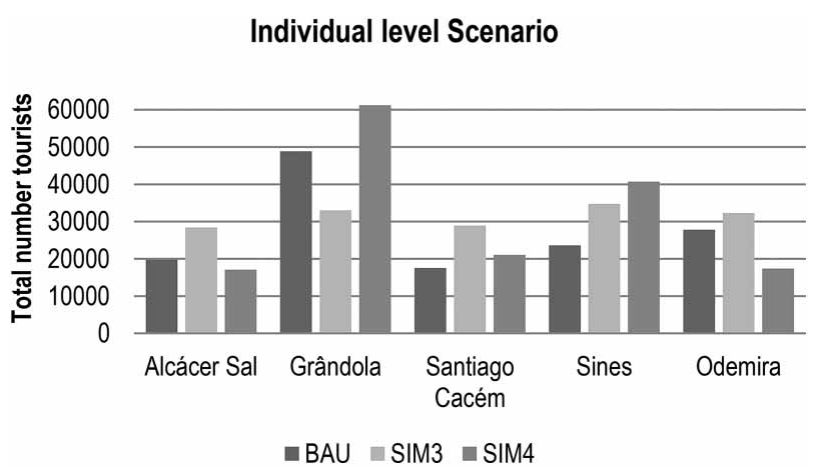

Figure 6. Total number of tourists in the individual motivation scenario.

satisfying there (reflecting its high occupancy rate), but when the satisfaction is not a criteria in the choice of the destination, tourists go to other destinations where there is supply for the type of activities they wish to pursue and according to their priority. For instance, if a sun and sea seeker goes to Grândola in the first time step because it is the best destination for sun and sea activities, when taking into account the priority for a destination in the next time step the same tourist will go to another destination with sun and sea activities, and so on, constituting a loop and ensuring the tourist agent goes through all the destinations during the simulation. All other destinations, which registered lower number of tourist than Grândola, verified an increase in tourist number, reflecting the priority and compatibility parameter influence regardless of occupancy and experience satisfaction.

The second simulation (Sim4) of the individual-level scenario explores the possible outcomes that the priority parameter may input in the ABM. We assume that the priority for a destination is a changing parameter that at each time step is updated; for example, when a tourist agent goes to a certain destination, in the next time step the priority for the destination will decrease. This generalization aims to investigate tourist behaviour at its simplest level assuming that this is the most basic way in which an agent can operate. The priority parameter was switch off to test the changes in distribution patterns produced. In Sim 4, 39\% tourists decide to go to Grândola, $26 \%$ to Sines, $13 \%$ to Santiago Cacém and $11 \%$ to Odemira and Alcácer do Sal (see Figure 6). Tourist concentration is dispersed throughout the destinations; still the majority go to Grândola because of the motivation compatibility and satisfaction related to highest occupancy.

Relating BAU to Sim4, the destinations that lost more tourists (Alcácer Sal and Odemira) are the ones that have lower occupancy rates; therefore, tourists also have a less satisfactory vacation and, since there is no priority for visiting the destinations, stop going there. All other destinations gain tourists without priority for visitation because of compatibility between supplied activities and tourist demand, occupancy rates and satisfaction of the experience.

Table 5. Change in tourist visitation (\%) between BAU-Sim3 and BAU-Sim4.

\begin{tabular}{llllll}
\hline Individual motivation scenario & Alcácer Sal & Grândola & Santiago Cacém & Sines & Odemira \\
\hline $\operatorname{Sim} 3$ & $30 \%$ & $-48 \%$ & $39 \%$ & $32 \%$ & $14 \%$ \\
$\operatorname{Sim} 4$ & $-16 \%$ & $20 \%$ & $17 \%$ & $42 \%$ & $-60 \%$ \\
\hline
\end{tabular}




\section{Discussion}

The ABM developed is a simplistic representation of the tourist decision-making process. Several assumptions made comprehend a great abstraction of the reality and require both theoretical and methodological deepening. The aim was to develop a theoretical proofof-concept ABM to explore, even with all the simplifications introduced, how tourists make decisions when selecting a destination to vacation. The ABM operates taking into account the social network and individual level, in order to represent and explore the basic theoretical principles of tourist decision-making behaviour (Bansal \& Eiselt, 2004; Cohen, Prayag, \& Moital, 2014; Kennedy, 2012). Tourist agents are endowed with rationality and their choices follow if-then rules imitating real-life behaviours; for example, the compatibility parameter is based on a mathematical match between tourists' motivation list and destinations' attraction list. They also have more abstract characteristics such as their social network influence and satisfaction of the vacation.

Two experiments were designed to explore how changes in the awareness for a destination and in tourist individual preferences change the destination selection. The scenarios tested point out that some relevant aspects such as the increase in awareness level of a destination make tourists' decisions more informed, so they are more likely to be satisfied with the vacation experience. The results also provide information about the individual preferences of the tourists, namely that priority, compatibility and satisfaction level from previous vacation experiences introduce different behaviour patterns. These behaviour patterns have been discussed by authors, such as Baggio (2008) and Mill and Morrison (2009), and are tested in the developed toy model. In fact, the model shows that tourists are pushed by their own internal forces such as motivation, priority of returning and satisfaction. There is indication that the number of previous satisfactory trips to a destination increases the probability of returning and fulfilling personal motivations. Also with the increase of awareness, or connectedness with the social network, tourists will take more informed decisions and they are more likely to choose a destination that meets the expectations, which leads to a satisfactory vacation. That is to say that the individual satisfaction and social network influence are intertwined in the way they influence the destination choice. Thus, we observe the way in which changes in the model parameters generate different patterns of visitation. These emergent patterns are driven by the interactions between tourists and with destinations, and by the feedback processes produced among them, which also infer with the destination decision-making process.

In future studies the aim is to increase the level of detail in the model. This toy model leads us to think that some parameters need refinement. For instance, in the individual preferences the motivation parameter will be divided by the type of activity and destination sought, not only because tourists sometimes choose destinations based on the type of activity but also because they really want to visit a specific place caring only afterwards about what activities they might engage in. The individual satisfaction will depend not only on the available accommodation space, and on some randomness that reflect other subjective and personal factors that influence tourists' satisfaction, but also on if what is expected is fulfilled.

In the social influence parameter, online networks with tourism information will be tested and eventually incorporated in the model, for example, tripadvisor and/or booking, in order to better represent how tourists' base their choices. We know online research is one of the main components in destination choice nowadays (Miguéns, Baggio, \& Costa, 2008). 
Other parameters, with geographical imprint, outside the scope of individual preferences and social network will be included in the model, for example, cost of the travel, seasonality and political strategies. The introduction and refinement of the model parameters, rules and information intend to represent the tourist decision-making process in a more realistic way.

\section{Concluding remarks}

In this paper, we explore the tourist individual decision-making process in selecting a vacation destination. A theoretical ABM was developed to try and replicate, in a simplified level, the tourist behaviour when evaluating and choosing destinations. A series of experiments were performed by building scenarios and changing model parameters to present results that reasonably reproduce real-world tourism system patterns. Even though the model presented is a basic representation with strongly simplified assumptions of the system processes, at the same time it contributes to the understanding of how tourism demand patterns are formed. An ABM is a tool that represents individual behaviour and interaction between agents, with simple if-then rules, producing simulations that resemble real-world patterns. With $\mathrm{ABM}$ it is possible to go beyond more traditional approaches to tourism research, identifying and addressing complex dynamics and processes that underlie the tourism system.

However, there are many challenges regarding the development of a tourism applied ABM, namely, deepening the understanding of the system dynamics, its components (specifically their motivations and behaviours) and how the network of interactions among them is placed. Also, with a deeper knowledge of the system comes the challenge of adapting more and more complex data on tourist characteristics and processes into model parameters and rules. The absence of standard validation procedures for ABM building raises issues regarding the level of reliability that researchers and stakeholders have in the model results. Nevertheless, once these challenges are overcome, ABM can be a useful tool for researchers in advancing tourism understanding and producing new insights about system dynamics. Equally for stakeholders these models can provide a toolbox for decision-making support, because they are a platform to explore from simple theoretical models that investigate hypothetical relationships, to empirical models that can replicate real-life problems. This way they are better equipped in their effort to understand the complex phenomenon in tourism destinations.

\section{Disclosure statement}

No potential conflict of interest was reported by the authors.

\section{Funding}

This work was supported by Fundação para a Ciência e a Tecnologia [grant number SFRH/BD/75984/ 2011].

\section{References}

Baggio, J. A., \& Baggio, R. (2009). Simulations and agent-based modelling (Dondena Working Papers No. 21). Università Bocconi, Milan, Italy.

Baggio, R. (2008). Symptoms of complexity in a tourism system. Tourism Analysis, 13(1), 1-20. 
Baggio, R., \& Klobas, J. (2011). Quantitative methods in tourism: A handbook (Vol. 48). Bristol: Channel View.

Baggio, R., \& Sainaghi, R. (2011). Complex and chaotic tourism systems: Towards a quantitative approach. International Journal of Contemporary Hospitality Management, 23(6), 840-861. doi:10.1108/09596111111153501

Bansal, H., \& Eiselt, H. A. (2004). Exploratory research of tourist motivations and planning. Tourism Management, 25(3), 387-396.

Batty, M. (1995). Planning support systems and the new logic of computation. Regional Development Dialogue, 16, 1-17.

Batty, M. (2008). The size, scale, and shape of cities. Science, 319(5864), 769-771.

Batty, M., Crooks, A. T., See, L. M., \& Heppenstall, A. J. (2012). Perspectives on agent-based models and geographical systems. In A. J. Heppenstall, A. T. Crooks, L. M. See, \& M. Batty (Eds.), Agent-based models of geographical systems (pp. 1-19). Dordrecht: Springer. doi:10.1007/ 978-90-481-8927-4

Bertalanffy, L. von. (1968). General system theory: Foundations, development, applications. New York, NY: Braziller.

Bonabeau, E. (2002). Agent-based modeling: Methods and techniques for simulating human systems. Proceedings of the National Academy of Sciences of the United States of America, 99(Suppl. 3), 7280-7287. doi:10.1073/pnas.082080899

Castle, C., \& Crooks, A. (2006). Principles and concepts of agent-based modelling for developing geospatial simulations (Working Paper 110). University College London, Centre for Advanced Spatial Analysis, London.

Cohen, S. A., Prayag, G., \& Moital, M. (2014). Consumer behaviour in tourism: Concepts, influences and opportunities. Current Issues in Tourism, 17(10), 872-909. doi:10.1080/13683500.2013. 850064

Couclelis, H. (2005). "Where has the future gone?" Rethinking the role of integrated land-use models in spatial planning. Environment and Planning A, 37(8), 1353-1371.

Crooks, A., Castle, C., \& Batty, M. (2008). Key challenges in agent-based modelling for geo-spatial simulation. Computers, Environment and Urban Systems, 32(6), 417-430. doi:10.1016/j. compenvurbsys.2008.09.004

Dann, G. (1977). Anomie, ego-enhancement and tourism. Annals of Tourism Research, 4(4), $184-194$.

Epstein, J. M. (1999). Agent-based computational models and generative social science. Generative Social Science: Studies in Agent-Based Computational Modeling, 4(5), 4-46.

Farrell, B. H., \& Twining-Ward, L. (2004). Reconceptualizing tourism. Annals of Tourism Research, 31(2), 274-295. doi:10.1016/j.annals.2003.12.002

Faulkner, B. (2000, August). "The future ain't what it used to be": Coping with change, turbulence and disasters in tourism research and destination management (Griffith University Public Lecture Series). Gold Coast, QLD.

Faulkner, B., \& Russell, R. (1997). Chaos and complexity in tourism: In search of a new perspective. Pacific Tourism Review, 1(2), 93-102.

Faulkner, B., Russell, R., Moscardo, G., \& Laws, E. (2001). Turbulence, chaos and complexity in tourism systems: A research direction for the new millennium. In B. Faulkner, G. Moscardo \& E. Laws (Eds.), Tourism and the 21st Century: Reflections on experience (pp. 328-349). London: Continuum Books.

Gilbert, N., \& Troitzsch, K. G. (2005). Simulation for the social scientist (2nd ed.). New York, NY: Open University Press.

Goossens, C. (2000). Tourism information and pleasure motivation. Annals of Tourism Research, $27(2), 301-321$.

Gunn, C. A. (1988). Tourism planning: Basics, concepts, cases. Washington: Taylor and Francis.

Horn, C. (2002). A complex systems perspective on communities and tourism: A comparison of two case studies in Kaikoura and Rotorua (Doctoral dissertation). Lincoln University, New Zealand.

Johnson, P. A., \& Sieber, R. E. (2009). Agent-based modelling: A dynamic scenario planning approach to tourism PSS. In S. Geertman \& J. Stillwell (Eds.), Planning support systems best practice and new methods (Vol. 95, pp. 221-226). Dordrecht: Springer.

Johnson, P. A., \& Sieber, R. E. (2010). An individual-based approach to modeling tourism dynamics. Tourism Analysis, 15(5), 517-530. 
Johnson, P. A., \& Sieber, R. E. (2011). Negotiating constraints to the adoption of agent-based modeling in tourism planning. Environment and Planning-Part B, 38(2), 307-321.

Kennedy, W. G. (2012). Modelling human behaviour in agent-based models. In E. A. J. Heppenstall, A. T. Crooks, L. M. See, \& M. Batty (Eds.), Agent-based models of geographical systems (pp. 167-179). Dordrecht: Springer. doi:10.1007/978-90-481-8927-4

Leiper, N. (1990). Tourist attraction systems. Annals of Tourism Research, 17(3), 367-384.

Lewin, R. (1999). Complexity: Life at the edge of chaos. London: A Pheonix Paperback.

Lorenz, E. (2000). The butterfly effect. Paper presented at 139th meeting of the American Association for the advancement of Science, Washington.

Macal, C. M., \& North, M. J. (2010). Tutorial on agent-based modelling and simulation. Journal of Simulation, 4(3), 151-162. doi:10.1057/jos.2010.3

Manson, S. M., Sun, S., \& Bonsal, D. (2012). Agent-based modeling and complexity. In E. A. J. Heppenstall, A. T. Crooks, L. M. See, \& M. Batty (Eds.), Agent-based models of geographical systems (pp. 125-139). Dordrecht: Springer. doi:10.1007/978-90-481-8927-4

McDonald, J. R. (2009). Complexity science: An alternative world view for understanding sustainable tourism development. Journal of Sustainable Tourism, 17(4), 455-471. doi:10.1080/ 09669580802495709

McKercher, B. (1999). A chaos approach to tourism. Tourism Management, 20(4), 425-434.

Miguéns, J., Baggio, R., \& Costa, C. (2008). Social media and tourism destinations: Tripadvisor case study. Paper presented at IASK ATR 2008 proceedings (Advances in Tourism Research 2008), Aveiro, Portugal. Retrieved from http://www.uib.es/depart/deeweb/pdi/acm/arxius/intermedia cio_tfg/baggio-aveiro2.pdf

Milgram, S. (1967). The small world problem. Psychology Today, 2(1), 60-67.

Mill, R. C., \& Morrison, A. M. (2009). The tourism system. Dubuque, IA: Kendall Hunt.

Parker, D. C., Manson, S. M., Janssen, M. A., Hoffmann, M. J., \& Deadman, P. (2003). Multi-agent systems for the simulation of land-use and land-cover change: A review. Annals of the Association of American Geographers, 93(2), 314-337. doi:10.1111/1467-8306.9302004

Pearce, D. (1989). Tourist development. Essex, UK: Longman.

Russell, R., \& Faulkner, B. (1999). Movers and shakers: Chaos makers in tourism development. Tourism Management, 20, 411-423.

Schelling, T. C. (1969). Models of segregation. The American Economic Review, 59(2), 488-493.

Scott, N., Cooper, C., \& Baggio, R. (2008). Destination networks. Annals of Tourism Research, 35(1), 169-188. doi:10.1016/j.annals.2007.07.004

Wu, F., \& Webster, C. J. (1998). Simulation of land development through the integration of cellular automata and multicriteria evaluation. Environment and Planning B, 25, 103-126.

Yoon, Y., \& Uysal, M. (2005). An examination of the effects of motivation and satisfaction on destination loyalty: A structural model. Tourism Management, 26(1), 45-56.

Zahra, A., \& Ryan, C. (2007). From chaos to cohesion - complexity in tourism structures: An analysis of New Zealand's regional tourism organizations. Tourism Management, 28(3), 854-862.

Zellner, M. L. (2008). Embracing complexity and uncertainty: The potential of agent-based modeling for environmental planning and policy. Planning Theory \& Practice, 9(4), 437-457. doi:10. $1080 / 14649350802481470$ 